\title{
Five-star rating system for acceptable quality and dose in CT
}

\author{
Mannudeep K. Kalra ${ }^{1} \cdot$ Madan M. Rehani ${ }^{1,2}$ (]) \\ Received: 13 April 2021 / Revised: 20 May 2021 / Accepted: 28 May 2021 / Published online: 11 June 2021 \\ (C) European Society of Radiology 2021
}

\section{Key Points}

- Recent papers have shown examples of the methodology involved in integrating image quality with radiation dose and assessing acceptable quality dose (AQD).

- As a further step in that direction, translating a 5-point score into a 5-star rating shall be helpful for wider and uniform application and shall be in line with the popular use of the 5-star rating.

Although awareness and monitoring of CT radiation doses have increased over the past two decades, there is very limited integration of diagnostic quality with radiation dose descriptors for purposes of $\mathrm{CT}$ protocol improvement and radiation dose optimization. Under the right clinical circumstances, CT provides clinically meaningful or treatment-altering information. Still, its use frequency, acquisition protocols (such as scanning in two or more phases), and high radiation doses (above recommended or reference dose levels) highlight a disturbing practice despite the availability of radiation dose monitoring with institutional or facility-specific commercial automatic dose monitoring software, national dose registries, and efforts around national and regional reference dose levels $[1,2]$.

Studies with large multi-institution or national registry suggest that despite recommendations for reduced dose CT protocols for specific clinical indications and availability of dose monitoring data, adoption of dose-conscious CT protocols remain low $[3,4]$. Over $65 \%$ of participating $\mathrm{CT}$ facilities in the USA exceeded the recommended radiation dose level for lung cancer screening with low-dose CT [3]. Likewise, only $8 \%$ of US CT facilities used low-dose CT for kidney stones despite recommendations and evidence within the American College of Radiology Appropriateness Criteria [4]. This pattern hints at a widespread disregard for sufficient diagnostic

Madan M. Rehani

madan.rehani@gmail.com; mrehani@mgh.harvard.edu

1 Massachusetts General Hospital, 55 Fruit Str, Boston, MA 02114, USA

2 Radiology Department, Massachusetts General Hospital, 175 Cambridge Str., Suite 244, Boston, MA 02114, USA quality at optimized or reduced radiation dose in favor of convenience, optimization, or medicolegal safety.

If the pre-dose monitoring era was emblematic of "can't fix what we don't know is broken" due to a lack of comprehensive dose data, is the current situation where a plethora of data on both recommended dose levels and monitoring services reflect "can't or won't fix what is not right" where dose reduction or optimization efforts do not synergize with traditional practices and comfort zones of many radiologists, physicists, and/or CT technologists? The situation calls for a black box, artificial intelligence (AI) models to take over the task of selecting the right dose for the right quality [5]. While that may be thought for future, recent studies have used subjective assessment on an institutional level so as to have an acceptable quality dose (AQD) [6, 7]. AQD can be personalized to body habitus, body region, scanner type, clinical indication, and quality and dose requirements. To be successful, AI models will need training datasets that remain scarce on what is adequate or just sufficient diagnostic quality for a wide range of variations in clinical indications, body regions, and patient body types. To account for inter-radiologist variations, one could use a set of "ground-truth" radiologists with pretraining in different aspects of diagnostic quality and radiation doses . Therefore, we propose a call to the radiology community to integrate diagnostic quality metrics into their dose data. While quantitative metrics and models of image quality exist, the jury remains split on the best metrics [8]. We propose a simple, 5-star rating system to motivate radiologists to take the initiative of ranking the diagnostic quality of each CT examination so that a databank of AQD can promote doseawareness and appropriate response to improve CT quality and safety. Such 5-star image quality ratings can allow CT facilities to compare themselves across scanners, clinical indications, and CT protocols within their facilities and outside 
practices. There are numerous precedents for such a 5-star rating in both imaging and non-imaging specialties of medicine and beyond medical practice. The United States Center for Medicare and Medicaid Services (CMS) employs a 5-Star Quality Rating system to rank nursing homes based on quality measures, staffing, and health inspection (assessment of practices that have or can cause actual harm to patients). There are parallels between the CMS systems and our proposed 5-star ranking system where both image quality/diagnostic confidence and radiation doses are assessed for minimizing potential of harm [9].

The 5-star rating for image quality (Tables 1 and 2) differs from previous linear, 3-point scoring for noise and acceptable spatial resolution (optimum; too much; too little) and 4-point scoring for diagnostic acceptability (1: fully acceptable; 2 : probably acceptable; 3 : acceptable only for limited conditions; 4: unacceptable) such as the one proposed as European Quality Criteria [10]. Conversion of a point scale to a 5-star rating goes with the intent of recent publications $[6,7]$. The non-linear 5-star rating of diagnostic quality rewards top rating to the clinical indication-based personalized protocols with "just adequate" diagnostic quality and, by extension, radiation doses such as low-dose CT for nodule follow-up, kidney stone evaluation, or paranasal sinus CT before an endoscopic surgery. CT with sufficient quality for routine or general-purpose $\mathrm{CT}$ protocols gets a less than perfect rating (four stars) to encourage indication-specific opportunities for protocol optimization. The rating system penalizes exams with diagnostic quality higher than needed (two stars) since it comes at the cost of an extra radiation dose. Exams with unacceptable diagnostic quality get the lowest rating (one star) since it leads to wasteful radiation and contrast usage and a delayed or missed care opportunity.

Mere local implementation of such a 5-star rating for diagnostic quality can be challenged due to substantial intra- and inter-radiologist variations both within a CT facility and across different facilities. To reduce such variations, we recommend the use of specific examples of clinical indications and CT images for each star rating. Another issue of such rating is the risk of a "pat on the back" approach since radiologists will rate according to their preferences and concern over missing unsuspected or subtle abnormalities, which can lead
Table 2 Proposed 5-star system for assessing diagnostic quality for the provided clinical reason or indication. (Key: CTDIvol, volume CT dose index; $D L P$, dose length product; \# Acceptable level of dose may be defined as local, regional, national, or international dose reference level). The proposed system is intended to help and not avoid or circumvent specific factors (scanner, protocol, patient, and/or practicerelated) responsible for low ratings

\begin{tabular}{|c|c|c|c|}
\hline Stars & Explanation & Radiation dose & Examples \\
\hline 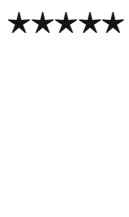 & $\begin{array}{l}\text { Images with some } \\
\text { level of noise } \\
\text { typical for low } \\
\text { dose CT for } \\
\text { specific clinical } \\
\text { indications }\end{array}$ & $\begin{array}{l}\text { CTDIvol and/or } \\
\text { DLP } \\
\text { well-optimized } \\
\text { locally and rea- } \\
\text { sonably below } \\
\text { reference levels }\end{array}$ & $\begin{array}{l}\text { Low-dose CT for } \\
\text { kidney stones }\end{array}$ \\
\hline 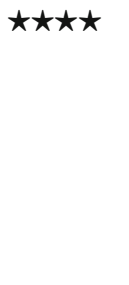 & $\begin{array}{l}\text { Adequate for most } \\
\text { routine or } \\
\text { general clinical } \\
\text { indications } \\
\text { where no special } \\
\text { consideration of } \\
\text { dose has } \\
\text { occurred }\end{array}$ & $\begin{array}{l}\text { CTDIvol and DLP } \\
\text { at reference } \\
\text { levels }{ }^{\#}\end{array}$ & $\begin{array}{l}\text { Routine abdomen } \\
\text { CT for cancer } \\
\text { staging }\end{array}$ \\
\hline$\star \star \star$ & $\begin{array}{l}\text { Evaluation limited } \\
\text { to some but not } \\
\text { all parts within a } \\
\text { region of } \\
\text { interest, does not } \\
\text { require repeat } \\
\text { scanning }\end{array}$ & $\begin{array}{l}\text { CTDIvol and DLP } \\
\text { at reference } \\
\text { levels }{ }^{\#}\end{array}$ & $\begin{array}{l}\text { CT with artifacts } \\
\text { over limited } \\
\text { parts of } \\
\text { anatomy } \\
\text { imaged }\end{array}$ \\
\hline$\star \star$ & $\begin{array}{l}\text { Higher than needed } \\
\text { quality (crisp } \\
\text { image) and } \\
\text { radiation dose } \\
\text { for a given } \\
\text { clinical } \\
\text { indication }\end{array}$ & $\begin{array}{l}\text { Higher than } \\
\text { needed or above } \\
\text { reference levels } \\
\text { CTDIvol and/or } \\
\text { DLP }{ }^{\#}\end{array}$ & $\begin{array}{l}\text { Lung nodule } \\
\text { follow-up CT } \\
\text { with routine } \\
\text { protocol/doses }\end{array}$ \\
\hline$\star$ & $\begin{array}{l}\text { Non-diagnostic for } \\
\text { required clinical } \\
\text { information, } \\
\text { often requires } \\
\text { repeat scanning }\end{array}$ & Undue dose & $\begin{array}{l}\text { Extremely high } \\
\text { noise or } \\
\text { artifacts images } \\
\text { or motion } \\
\text { impaired CT } \\
\text { for cancer } \\
\text { staging }\end{array}$ \\
\hline
\end{tabular}

to a 4- or 5-star rating to most CT exams despite a higher than needed diagnostic quality. Collected and compared over multiple CT facilities, such a system can provide invaluable
Table 1 Five-star system for the diagnostic quality of CT images

\begin{tabular}{ll}
\hline Stars & Diagnostic quality in consideration of dose \\
\hline$\star \star \star \star \star$ & Acceptable, despite high image noise associated with low dose CT \\
$\star \star \star \star$ & Acceptable for all parts within the region of interest \\
$\star \star \star$ & $\begin{array}{c}\text { Acceptable, despite unintended high noise and/or artifacts in some parts (evaluation limited to } \\
\text { certain parts of the anatomy scanned) }\end{array}$ \\
$\star \star$ & Excellent quality with unjustifiably low image noise and no artifacts \\
$\star$ & Non-diagnostic (excessive noise/artifacts, poor contrast) \\
\hline
\end{tabular}


insight into how radiologists perceive diagnostic quality at different dose levels, which can help device-specific training programs at outlying CT facilities. Such data can also help target dose reduction efforts while re-training radiologists on what constitutes a 5-star diagnostic quality. We should integrate the diagnostic quality scores within the dose monitoring tools along with information on specific clinical reasons for individual CT examinations for a meaningful impact. Finally, the intent of our proposed 5-star rating system is to help raise awareness and to guide appropriate investigations into radiation dose, patient, and image quality attributes responsible for low ratings.

In conclusion, we believe that incorporating the 5-star ratings within the radiology information system (PACS or dictation system) should not add more than a few seconds for most cases. Such a 5-star rating can provide tremendous resources for reduction or optimization of radiation dose while making diagnostic quality clinical indications specific.

Funding The authors state that this work has not received any funding.

\section{Declarations}

Guarantor The scientific guarantor of this publication is Manudeep Kalra.

Conflict of interest Manudeep Kalra and Madan Rehani declare no conflict of interest.

\section{Statistics and biometry Not involved}

Informed consent Written informed consent is not required because this is only an opinion article involving no data collection on human subjects or Protected Health Information (PHI).

Ethical approval Institutional Review Board approval is not required because the study does not include any human subjects or Protected Health Information (PHI).

\section{Methodology}

- Editorial

\section{References}

1. Tsalafoutas IA, Hassan Kharita M, Al-Naemi H, Kalra MK (2020) Radiation dose monitoring in computed tomography: status, options and limitations. Phys Med 79:1-15. https://doi.org/10.1016/ j.ejmp.2020.08.020

2. Homayounieh F, Holmberg O, Al Umairi R et al (2020) Variations in CT utilization, protocols, and radiation doses in COVID-19 pneumonia: results from 28 countries in the IAEA study. Radiology 203453. https://doi.org/10.1148/radiol.2020203453

3. Weisenthal K, Karthik P, Shaw M et al (2018) Evaluation of kidney stones with reduced-radiation dose CT: progress from 2011-2012 to 2015-2016-not there yet. Radiology 286:581-589. https://doi.org/ 10.1148/radiol.2017170285

4. Demb J, Chu P, Yu S et al (2019) Analysis of computed tomography radiation doses used for lung cancer screening scans. JAMA Intern Med 179:1650-1657. https://doi.org/10.1001/ jamainternmed.2019.3893

5. Ma JJ, Nakarmi U, Kin CYS et al (2020) Diagnostic image quality assessment and classification in medical imaging: opportunities and challenges. Proc IEEE Int Symp Biomed Imaging 2020:337-340. https://doi.org/10.1109/isbi45749.2020.9098735

6. Padole AM, Sagar P, Westra SJ et al (2019) Development and validation of image quality scoring criteria (IQSC) for pediatric CT: a preliminary study. Insights Imaging 10:95. https://doi.org/ 10.1186/s13244-019-0769-8

7. Kharita MH, AlNaemi H, Kini V, Alkhazzam S, Rehani MM (2020) Development of image quality related reference doses called acceptable quality doses (AQD) in paediatric CT exams in Qatar. Eur Radiol. https://doi.org/10.1007/s00330-020-07375-7

8. Verdun FR, Racine D, Ott JG et al (2015) Image quality in CT: from physical measurements to model observers. Phys Med 31: 823-843. https://doi.org/10.1016/j.ejmp.2015.08.007

9. Mukamel DB, Weimer DL, Shi Y, Ladd H, Saliba D (2020) Comparison of consumer rankings with centers for Medicare \& Medicaid services five-star rankings of nursing homes. JAMA Netw Open 3:e204798. https://doi.org/10.1001/jamanetworkopen. 2020.4798

10. European Guidelines on Quality Criteria for computed tomography. https://op.europa.eu/en/publication-detail/-/publication/d229c9e1a967-49de-b169-59ee68605fla. Accessed on April 1, 2021

Publisher's note Springer Nature remains neutral with regard to jurisdictional claims in published maps and institutional affiliations. 